\title{
EDITORIAL
}

\section{Whither pediatric physician-scientist training in the COVID-19}

\section{era}

Pediatric Research (2021) 89:1041-1042; https://doi.org/10.1038/s41390020-1026-z

Pediatric physician-scientists play a critical role in translating research findings from basic and clinical research into new paradigms and approaches pertinent to our care for children. The United States has long been a leader in biomedical research; however, this workforce has been diminishing over many years secondary to decreases in recruitment, limited funding and protected time, and attrition during training. ${ }^{1}$ This pipeline of pediatric physician-scientists is critical not only for discovery but also for contextualizing specific findings and extrapolating those findings to understanding development and treatment strategies that can reduce disease burden and improve quality of life.

The National Institutes of Health (NIH), American Board of Pediatrics ( $A B P$ ), and other institutions heavily involved in the pediatric research community have expressed concern regarding the reduction of trainees in this pipeline. In addition to integrated and accelerated research pathways during residency, a number of early and mid-career training programs focus on improving the environment for physician-scientist development and success. ${ }^{2}$ These provide funding and mentoring to enable successful transition of trainees to become independent scientists. While other fields or specialties are dealing with similar issues with recruitment and successful training, this still presents a career path with significant obstacles, especially in regard to training pediatricians. $^{3}$ Specifically, the lack of consistent, structured training programs that provide adequate protected time and facilities/space to perform research represents a significant obstacle to interested or curious trainees. In turn, this may limit the number of trainees who decide to pursue a career in pediatrics, where clinical requirements and scheduling during residency may be too stringent compared with other specialties.

Now, the coronavirus disease 2019 (COVID-19) pandemic has required many research endeavors to come to a halt. Depending on the guidelines of specific institutions, critical or essential projects may continue, but where that line between essential and nonessential activities is drawn is ambiguous and constantly changing. This may also place "essential" workers, in this case researchers at all levels, at risk if adequate precautions are not taken. As we enter different phases in our return to work, it is very likely that continued isolation, distancing, and appropriate precautions related to masking/hygiene will need to continue for an indefinite amount of time to prioritize safety. Thus, opening up research facilities and workspaces will not necessarily mean returning to former modi operandi.

A potential and realistic plan to restart or accelerate research will involve decreasing the number of individuals in a lab or workspace at the same time and shifting working hours for some to limit proximate exposure. It will likely also include limiting access to shared or core facilities. The obvious downside to this reasonable approach is that it will place significant limits in training, specifically in regards to experience and education, and increase the barriers to success for trainees and many early and mid-stage investigators. Early or less well-funded researchers may be more dependent on these shared or core facilities; they may also have access to fewer desks or lab benches in which to spread the work. This means that, unintentionally, these investigators may have less access to perform their research compared to others, resulting in longer times, with increased costs, related to their studies and slower output of results.

The effects of COVID-19 clearly demonstrate how different groups of individuals are more or less vulnerable, not just to infection, but to the downstream effects of our response to the pandemic. ${ }^{4}$ In addition to creating more barriers to achieving the markers of success in a specific time frame, including publications, grants, presentations at national and local conferences and seminars, additional barriers may limit the decision or ability to pursue a career as a physician-scientist. These include issues with child care or elder care, home or work environments that represent a significant burden on individual or family health and safety, travel restrictions interfering with collaborations, and negative impacts on laboratory materials and equipment supply chains. While a significant discrepancy in regards to minority or gender representation in science is well recognized, COVID-19 may disproportionately affect underrepresented groups in the ability to conduct research and reach the next stages in their career. ${ }^{5}$

In the short term, the significant financial impact of shelteringin-place and limiting nonessential care and business has led to hiring freezes, job loss, and/or pay cuts at many institutions. Potential loss or limitations in the use of laboratory assistants will also negatively influence the outcomes for these individuals. For trainees nearing the end of their fellowship or post-doctoral training, the changes in laboratory finances and personnel will obviously effect not only their ability to find a job and begin a career, but also their clinical expectations and protected research time to initiate or continue projects. In the long term, a decrease in protected research time over a number of months to years will significantly hinder their ability to successfully publish, write grants, or complete training within a specified period of time. This potential lack of productivity may not reflect well on an applicant's potential for academic success when applying for a faculty position or competitive grant, and overall could interfere with the individual's precious career development timeline.

While many of these concerns regarding barriers to recruiting and adequately training pediatric physician-scientists existed before COVID-19, the pandemic and our response to it will exacerbate the underlying problems for many years. We need to be conscious of these issues and actively work to support trainees. This will require a concerted commitment from seasoned investigators, educators, mentors, and administrators to identify and address these barriers for training future physician-scientists. We will need to accommodate new requirements for trainees and their time and restrictions to their education, providing adequate 
1042

space and resources to perform research, while still making every effort to protect their time. The $\mathrm{NIH} \mathrm{K}$ funding mechanism is an important mechanism for early investigators to transition from trainee to early faculty. Many trainees are in specific fellowship programs that are funded for a specific length of time. Funding agencies, including the $\mathrm{NIH}$, will need to alter expectations of new applicants and how grant applications are evaluated, including their previous expectations for number of publications, preliminary data, or productivity; our leading medical organizations should advocate that Congress increase funding of our basic research infrastructure including the physician-scientist pipeline. Stronger consideration of the impact of new limitations and guidelines on underrepresented groups will be essential for developing a diverse community of researchers. This will be essential to increase the quality of research that will broadly impact not just our understanding of growth, development, and disease processes, but improve both short- and long-term outcomes of physician-scientist training programs.

\section{ACKNOWLEDGEMENTS}

F.F.G. is supported by NIH R01NS107039, U01NS092764, and R35NS097299.

\section{AUTHOR CONTRIBUTIONS}

F.F.G. and M.B.H. conceptualized and collaborated on the submitted manuscript. F.F.G. and M.B.H. approved the final manuscript as submitted and agree to be accountable for all aspects of the work

\section{ADDITIONAL INFORMATION}

Competing interests: The authors declare no competing interests.

Publisher's note Springer Nature remains neutral with regard to jurisdictional claims in published maps and institutional affiliations.

Fernando F. Gonzalez ${ }^{1}$ and Melvin B. Heyman ${ }^{1}$ ${ }^{1}$ Department of Pediatrics, University of California, San Francisco,

CA, USA

Correspondence: Fernando F. Gonzalez

(Fernando.Gonzalez@ucsf.edu)

\section{REFERENCES}

1. Burns, A. M. et al. Fixing the leaky pipeline: identifying solutions for improving pediatrician-scientist training during pediatric residency. Pediatr. Res. (2020). https://doi.org/10.1038/s41390-020-0837-2.

2. Daye, D., Patel, C. B., Ahn, J. \& Nguyen, F. T. Challenges and opportunities for reinvigorating the physician-scientist pipeline. J. Clin. Invest. 125, 883-887 (2015).

3. Heyman, M. B. et al. Challenges of funding pediatric fellowship programs-invited commentary from the Council of Pediatric Subspecialties. J. Pediatr. 204, 4-6.e1 (2019).

4. CDC. COVID-19 in racial and ethnic minority groups [Internet]. https://www.cdc. gov/coronavirus/2019-ncov/need-extra-precautions/racial-ethnic-minorities.html (2020).

5. Kwan, J. M. et al. Exploring intentions of physician-scientist trainees: factors influencing $\mathrm{MD}$ and $\mathrm{MD} / \mathrm{PhD}$ interest in research careers. BMC Med. Educ. 17, 115 (2017). 